\title{
Ações do enfermeiro no pré-natal e a importância atribuída pelas gestantes
}

\author{
Ernandes Gonçalves Dias \\ Enfermeiro Especialista em Enfermagem do trabalho e Docência na Saúde. Mestrando do Programa de \\ Inovação e Tecnologia em Enfermagem na Universidade de São Paulo (EERP-USP). \\ Docente na Faculdade Presidente Antônio Carlos de Porteirinha e Faculdade Verde Norte (FAVENORTE). \\ $\square$ nandesenf@usp.br \\ Gisele Brito dos Anjos \\ Graduanda em Enfermagem. Faculdade Presidente Antônio Carlos de Porteirinha. \\ $\triangle$ giseleanjos.90@gmail.com \\ Luciene Alves \\ Graduanda em Enfermagem. Faculdade Presidente Antônio Carlos de Porteirinha. \\ $\square$ luciene.lecy@gmail.com \\ Sayonara Nayranne Pereira \\ Graduanda em Enfermagem. Faculdade Presidente Antônio Carlos de Porteirinha. \\ $\triangle$ sayonara.nayranne@yahoo.com

\section{Lyliane Martins Campos} \\ Enfermeira Especialista em Docência na Saúde. \\ Docente na Faculdade Presidente Antônio Carlos de Porteirinha e Faculdade Verde Norte (FAVENORTE). \\ $\triangle$ lyliport@gmail.com
}

Recebido em 12 de dezembro de 2017

Aceito em 24 de maio de 2018

\section{Resumo:}

O pré-natal é essencial para que a mulher se prepare para ser mãe, e é por meio das consultas e outras ações desenvolvidas no âmbito da Estratégia Saúde da Família que a gestante é acompanhada quanto ao desenvolvimento de sua gestação e as condições do bebê. Objetivou-se identificar a importância atribuída pelas gestantes às ações do enfermeiro no pré-natal. Trata-se de um estudo descritivo de natureza qualitativa, realizado com 13 gestantes da Estratégia de Saúde da Família Vila Serranópolis. Os dados foram coletados no período de março a abril de 2016 por meio de uma entrevista estruturada. Os resultados mostraram que as gestantes identificaram a mensuração de dados vitais, as orientações e as reuniões educativas como ações da assistência pré-natal realizadas pelo enfermeiro e reconheceram a importância destas. Consideraram que os conselhos, esclarecimento de dúvidas e a tranquilidade transmitida pelos enfermeiros durante as consultas do pré-natal como fundamentais para o desenvolvimento da gestação. Conclui-se que apesar das gestantes reconhecerem a importância das ações realizadas pelo enfermeiro durante o pré-natal é necessário que esses profissionais busquem estratégias para melhorar a assistência às gestantes no sentido de reforçar as ações de educação em saúde e a criação de vínculo entre à gestante e o serviço de saúde.

Palavras-chave: Gestantes, Cuidado Pré-Natal, Enfermagem Obstétrica. 


\title{
Nurses' actions in prenatal care and the importance attributed by pregnant women
}

\begin{abstract}
:
Prenatal care is essential for the woman to prepare herself to be a mother, and it is through the consultations and other actions developed within the scope of the Family Health Strategy that the pregnant woman is monitored regarding the development of her gestation and the conditions of the baby. The objective was to identify the importance attributed by the pregnant women to the actions of the nurse in prenatal care. This is a descriptive study of a qualitative nature, carried out with 13 pregnant women from the Family Health Strategy Vila Serranópolis. Data were collected from March to April 2016 through a structured interview. The results showed that the pregnant women identified the measurement of vital data, orientations and educational meetings as prenatal care actions performed by the nurse and recognized the importance of these. They considered the advice, clarification of doubts and the tranquility transmitted by the nurses during the prenatal consultations as fundamental for the development of gestation. It is concluded that although the pregnant women recognize the importance of the actions performed by the nurse during prenatal care, it is necessary that these professionals seek strategies to improve the assistance to pregnant women in order to reinforce the actions of health education and the creation of a link between the pregnant woman and the health service.
\end{abstract}

Keywords: Pregnant Women, Prenatal Care, Obstetric Nursing.

\section{Acciones del enfermero en el prénatal y la importancia atribuida por los gestantes}

\section{Resumen:}

El prenatal es esencial para que la mujer se prepare para ser madre, y es por medio de las consultas y otras acciones desarrolladas en el marco de la Estrategia Salud de la Familia que la gestante va acompañada en el desarrollo de su gestación y las condiciones del bebé. Se objetivó identificar la importancia atribuida por las gestantes a las acciones del enfermero en el prenatal. Se trata de un estudio descriptivo de naturaleza cualitativa, realizado con 13 gestantes de la Estrategia de Salud de la Familia Vila Serranópolis. Los datos fueron recolectados en el período de marzo a abril de 2016 por medio de una entrevista estructurada. Los resultados mostraron que las gestantes identificaron la medición de datos vitales, las orientaciones y las reuniones educativas como acciones de la asistencia prenatal realizadas por el enfermero y reconocieron la importancia de éstas. Consideraron que los consejos, aclaración de dudas y la tranquilidad transmitida por los enfermeros durante las consultas del prenatal como fundamentales para el desarrollo de la gestación. Se concluye que a pesar de las gestantes reconocer la importancia de las acciones realizadas por el enfermero durante el prenatal es necesario que estos profesionales busquen estrategias para mejorar la asistencia a las gestantes en el sentido de reforzar las acciones de educación en salud y la creación de vínculo entre la salud gestante y el servicio de salud.

Palabras clave: Mujeres Embarazadas, Atención Prenatal, Enfermería Obstétrica.

\section{INTRODUÇÃO}

Segundo Vieira e Parizotto (2013) a gestação é um período de mudanças na vida da mulher, entre elas físicas, psicológicas e sociais. A mulher pode se torna mais sensível e 
emotiva nesta fase da vida e precisa receber orientações eficientes para tornar o período gravídico mais tranquilo para si e para a família.

Para Martins et al. (2012) o pré-natal é essencial para que a mulher se prepare para ser mãe, e é por meio das consultas e outras ações desenvolvidas no âmbito da Estratégia Saúde da Família (ESF) que a gestante é acompanhada quanto ao desenvolvimento de sua gestação e as condições do bebê. Dessa forma, a assistência da equipe de saúde pode ser considerada como uma ferramenta para a prevenção de complicações clínicas e obstétricas no decorrer da gestação e parto.

No pré-natal a gestante é acolhida e conduzida por meio da assistência de uma equipe multiprofissional de saúde, que realiza ações que visam prepará-la para vivenciar a gestação e o parto com tranquilidade e saúde (DIAS et al., 2015a).

Conforme Cunha et al. (2009) a atuação do enfermeiro é importante no pré-natal, assim, é necessário que este profissional seja qualificado para atender as necessidades da mulher durante o ciclo gravídico-puerperal com conhecimentos adequados e atualizados, de forma a oferecer uma assistência eficaz.

Os profissionais de enfermagem desempenham uma função fundamental em relação à orientação na consulta da gestante no pré-natal, assim sana as dúvidas, mantêm a mulher orientada quanto à importância das consultas e exames necessários na gestação. Neste sentido, o enfermeiro precisa realizar ações de maneira eficaz, resguardando a gestante de negligências, imperícias e imprudências, atuando de forma ética e responsável, para assegurar o nascimento de um concepto saudável (LEMES, 2012).

Diante dessas considerações, acredita-se que as ações do enfermeiro são importantes no pré-natal, uma vez que por meio da assistência prestada, é possível identificar intercorrências precocemente e monitorar as gestantes que se encontram em situações de riscos. Além disso, as gestantes podem se sentir mais acolhidas diante das descobertas advindas em cada semana de gestação, proporcionando assim, uma gravidez mais segura.

Assim, este estudo teve como objetivo identificar a importância atribuída pelas gestantes às ações do enfermeiro no pré-natal e responder ao questionamento norteador deste estudo: qual a importância que as gestantes atribuem às ações realizadas pelos enfermeiros durante o pré-natal? 


\section{MATERIAL E MÉTODOS}

Trata-se de um estudo descritivo de natureza qualitativa, realizado com 13 gestantes cadastradas na ESF Vila Serranópolis, da cidade de Porteirinha, Minas Gerais. Foram inclusas as gestantes da área de adscrição da Unidade Básica de Saúde (UBS), cadastradas no Sisprenatal com idade igual ou superior a 18 anos.

Todos os caminhos metodológicos deste estudo obedeceram às normas estabelecidas pela Resolução 466/12 do Conselho Nacional de Saúde que dispõe sobre pesquisa envolvendo seres humanos. Dessa forma, cada que consentiu sua participação no estudo assinou um Termo de Consentimento Livre e Esclarecido, autorizando o uso dos dados fornecidos aos pesquisadores. O projeto desse estudo foi submetido à avaliação do Comitê de Ética em Pesquisa da Universidade Presidente Antônio Carlos e aprovado pelo Parecer Consubstanciado no 1.445 .566 em 10 de março de 2016.

Os dados foram coletados no período de março a abril de 2016, por meio de uma entrevista estruturada composta de questões objetivas, que caracterizaram as gestantes, e subjetivas que buscaram identificar a visão das gestantes sobre a importância das ações realizadas pelo enfermeiro durante o pré-natal. O roteiro de entrevista era composto pelas questões: "Que atividades o enfermeiro realiza durante seu atendimento de pré-natal?", "O que o enfermeiro faz na rotina de acompanhamento de sua gestação?", "O que você pensa sobre essas ações?”, “Qual importância dessas ações para sua gestação e pré-natal”.

A coleta dos dados foi realizada pelos próprios pesquisadores, na ESF Vila Serranópolis, logo após a gestante terminar a consulta médica ou de enfermagem. Havia também a possibilidade de a gestante agendar visita do pesquisador em sua residência para submissão à entrevista, se fosse de sua preferência. Durante a coleta foi usado um gravador de áudio para o registro da entrevista que teve duração média de 20 minutos. Os dados foram coletados até o momento em que os pesquisadores observaram saturação nos discursos, pela repetição de informações nos relatos das gestantes.

Para análise dos dados, as entrevistas foram transcritas na íntegra, realizou-se leituras sucessivas dos depoimentos das gestantes a fim de categorizá-los e estes foram analisados de acordo Análise do Conteúdo na perspectiva de Bardin (2009). Assim, emergiram a caracterização das gestantes e as categorias de análise "ações realizadas pelos enfermeiros no 
pré-natal" e "importância das ações realizadas pelo enfermeiro no pré-natal”. A identidade das entrevistadas foi resguarda pela substituição de seus nomes por nomes de cidades do norte de Minas.

\section{RESULTADOS E DISCUSSÃO}

\section{Caracterização das participantes}

As gestantes deste estudo foram caracterizadas em relação às variáveis, cor, idade, escolaridade, estado civil, renda e núcleo de convívio familiar. Em relação à cor a maioria das gestantes se autodeclararam pardas, a idade variou entre 19 e 38 anos, sendo que a maior parte tinha entre 31 e 38 anos. O estado civil variou entre gestantes casadas, solteiras, divorciadas e em união estável.

Observou-se baixa escolaridade entre as gestantes, a maior parte tinha o ensino médio completo. A renda familiar declarada variou de um a três salários mínimos incompletos, sendo que a maior parte mora com quatro ou mais pessoas no lar, donde se compreende haver baixa renda per capta.

\section{Ações realizadas pelo enfermeiro no pré-natal}

Nas entrevistas com as gestantes emergiram as ações que o enfermeiro realiza no prénatal. As gestantes reconhecem como ações do pré-natal, as consultas de enfermagem, registros das informações nos impressos, medidas antropométricas, solicitação de exames, aferição de dados vitais, orientações e reuniões educativas, como se observa nos relatos das entrevistadas abaixo:

\footnotetext{
“Ah eu só fiz uma consulta só. A enfermeira só preencheu a cadernetinha, preencheu a ficha para exame de HIV e conversou comigo e deu algumas dicas, algumas coisas assim só." (Janaúba).

"Pesagem e a pressão, afere a pressão, ai vai dando andamento, o peso e a pressão se tá certinho, né.” (Monte Azul).

“Dá orientações, e geralmente ela orienta né, as coisas, alguns exames tudo certinho. Muito importante tem que ter a orientações, pra gente que não sabe, esclarecer as dúvidas." (Porteirinha).
} 


\begin{abstract}
“Fazer a pesagem né, medir altura, pressão, anotar no cartão e orientações também [...]” (Mato Verde).

“Tipo assim, teve é reunião, mediu minha pressão, e me avaliou no caso [...]" (Pai Pedro).
\end{abstract}

Conforme Andrade et al. (2015) o enfermeiro precisa realizar consulta de pré-natal humanizada e qualificada. A consulta de pré-natal qualificada deve seguir um roteiro básico, com atendimento aos aspectos sociais, epidemiológicos, antecedentes pessoais, ginecológico, sexuais e obstétricos e dados sobre a gestação atual. 0 enfermeiro pode solicitar exames e encaminhar a gestante para outros profissionais da saúde para que o acompanhamento seja processado de forma integral.

A atenção humanização é necessária como meio de resgate ao respeito no atendimento à gestante, assim, é importante que prestar cuidado humanizado seja uma finalidade de toda a equipe de enfermagem. No período gestacional, o enfermeiro precisa enfatizar as orientações às gestantes, por meio da educação em saúde, de maneira que estejam claras as alterações ocorridas no corpo e mente em função da gravidez. Sabe-se que este é um período delicado, onde há por parte da maioria das mulheres e da família uma grande expectativa em torno da gestação (NUNES; SILVA, 2012).

Os registros das consultas na caderneta da gestante é uma ação importante para a assistência ao pré-natal nos serviços de saúde, para que o cuidado seja contínuo, com completo histórico da gestação, e assistência ao parto, promovendo a melhor tomada de decisão em situações adversas (SALES; SEMENTE; FERNANDES, 2013).

Segundo Niquini et al. (2012) a coleta dos dados vitais, das medidas antropométricas e o registros dos mesmos durante o pré-natal pode assegurar a qualidade da assistência além de dar andamento a atenção à gestante.

O acompanhamento contínuo do pré-natal assegura a mãe e ao bebê uma gestação e parto saudáveis. É essencial que sejam promovidas ações de promoção e prevenção contra a morbimortalidade infantil e materna (FREITAS et al. 2010).

Para Paris, Pelloso e Martins (2013) os exames laboratoriais de rotina no pré-natal, são úteis para identificar precocemente morbidades gestacionais, para tratá-las, prevenir a 
infecção fetal e óbitos da gestante, do concepto e do recém-nascido. Os resultados dos exames laboratoriais possibilitam realizar monitoramento para a classificação do risco pré-natal.

De acordo Sousa, Mendonça e Torres (2012) é na consulta do pré-natal que o enfermeiro tem a oportunidade de manter o acompanhamento da gestante, com anotações essenciais do atendimento realizado. Nesse sentido, o enfermeiro realiza medidas de promoção e prevenção à saúde da mulher e do feto, tais como aferição da pressão arterial, peso, altura, etc. Como meio de assegurar que todos os registros sejam respaldados, os mesmos precisam estar também no prontuário da gestante.

Para Marcon, Roecker e Souza (2010) as reuniões educativas com as gestantes possibilita ao enfermeiro transmitir conhecimento acerca do processo gestacional e utilizar dessa ferramenta como meio de promoção da saúde. Acredita-se que tais ações aproximam as gestantes com os serviços de saúde, e que assim seja alcançada melhoria na qualidade da atenção primária, influenciando de maneira positiva nos indicadores de morbimortalidade materno-infantil.

\section{Importância das ações realizadas pelo enfermeiro no pré-natal}

As gestantes relataram sobre a importância das ações realizadas pelo enfermeiro no pré-natal. As participantes reconhecem a importância das orientações, das dicas, esclarecimento de dúvidas e apontam o enfermeiro como o profissional que estabelece o primeiro contato com a gestante e lhes transfere tranquilidade. Os discursos da importância atribuída às ações do enfermeiro estão implicitamente direcionados às ações de educação em saúde realizadas por este profissional, conforme mostra os discursos a seguir:

"[...] você começa a conhecer mais, até porque igual, por exemplo, é a primeira gravidez minha. Eu não sei nada, não entendo nada. Então assim, com o que ela fala, com as dicas que ela dá, tira as dúvidas, isso é o que ela ensina." (Janaúba).

“[...] ah eu acho essencial o papel do enfermeiro demais, porque assim às vezes você chega aqui com algum probleminha o médico não tá, ela te passa tranquilidade te instrui direitinho o que você deve fazer ou não, é muito importante." (Espinosa).

“[...] são coisas assim pra mim que ajuda, de uma certa forma ajuda, que eu tive muitas dúvidas na outra gestação que foram esclarecidas nessa, que eu procuro não cometer os mesmos erros, que eu cometi na outra." (Montes Claros). 
“[...] no meu caso eu tem 12 anos que engravidei, assim né, na gestação passada com essa totalmente diferente, então eu preciso deles pra poder tirar minhas dúvidas e cada ano passa vai renovando. Quando ganhei minha menina foi diferente. Agora nossa! Tudo mudou." (Catuti).

"O enfermeiro que inicia tudo, ele que vai orientar, fazer orientações, ele vai tá dando andamento no pré-natal pra depois ir nas consultas médicas." (Monte Azul).

Segundo Souza, Bernardo e Santana (2013) o enfermeiro é importante na assistência durante o pré-natal na ESF. Em concordância ao autor supracitado, Carvalho (2010) relata que o bom desempenho do enfermeiro é fundamental para que a gestante se sinta satisfeita e crie elo com o profissional, possibilitando um relacionamento de confiança entre ambas as partes.

Para Dias et al. (2015b) as gestantes entendem que é importante adquirir conhecimentos durante o pré-natal para que seja assegurado uma gestação, parto e puerpério sem complicações. Deste modo é fundamental que o enfermeiro seja habilitado para prestar uma assistência eficaz e contínua junto à gestante, intensificando a implementação de ações educativas, visando garantir uma assistência completa e contínua ao longo de todo período gestacional.

Conforme Martinelli et al. (2014) as gestantes aderem aos serviços e buscam os profissionais de saúde a partir do momento em que ela recebe um atendimento adequado e de qualidade, onde se sinta acolhida. As ações realizadas no pré-natal são importantes para uma assistência humanizada na saúde obstétrica.

Segundo Silva, Andrade e Bosi (2014) o acolhimento, o trabalho de educação em saúde e o cuidado humanizado na UBS garantem uma relação baseada no diálogo entre os profissionais e usuárias, portanto, são fundamentais para uma assistência de qualidade para as gestantes.

Apesar da maioria das gestantes compreenderem a importância das ações realizadas pelo enfermeiro durante o pré-natal, uma entrevistada não considerou relevantes as ações realizadas, como mostra o discurso a seguir:

"Não acho muito não, sei lá, todas as consultas não é necessário fazer isso [...]." (Gameleiras). 
Segundo Araújo et al. (2010b) ainda que o pré-natal seja prioridade para o Ministério da Saúde é também atividade dos enfermeiros na ESF, existe problemas para que as mulheres cheguem na Unidade de Saúde, e os profissionais enfermeiros nem sempre atendem as consultas de forma certa, o que causa uma deficiência na qualidade do atendimento.

De acordo Carrara e Oliveira (2013) a satisfação das gestantes se dá por meio da qualidade do atendimento realizado pelos enfermeiros. É por meio dos esclarecimentos de dúvidas, das orientações dadas que o enfermeiro pode fazer a diferença na vida da mulher e que esta pode compreender a importância da assistência de enfermagem no pré-natal.

Assim, Spindola, Progianti e Penna (2012) afirmam que o diferencial da consulta de enfermagem está relacionada a escuta atenta do enfermeiro, além de ser também um momento que a gestante tem espaço para sanar dúvidas, e o enfermeiro estabelecer vínculo. Para Moreira, Carvalho e Ribeiro (2012) a assistência que o enfermeiro dispensa às mulheres no pré-natal faz com que as gestantes tenham liberdade para se expressar, dessa forma, torna a consulta de enfermagem mais efetiva em relação ao acompanhamento da maioria dos outros profissionais que assistem a mulher na gestação, em especial, o médico.

\section{CONSIDERAÇÕES FINAIS}

Quanto às ações realizadas pelo enfermeiro as gestantes demonstraram conhecê-las, e as consideraram relevante, o que torna possível a adesão ao pré-natal e possibilita ao profissional de enfermagem prestar uma assistência de qualidade. Dessa forma é fundamental a continuidade dessas ações durante as consultas de enfermagem para que as gestantes tenham um acompanhamento de qualidade e sejam assistidas ao longo de todo período gravídico.

As gestantes consideram fundamental para o desenvolvimento da gestação os conselhos, esclarecimento de dúvidas e a tranquilidade transmitida pelos profissionais enfermeiros durante as consultas de enfermagem de pré-natal. Neste sentido faz se necessário ressaltar que o enfermeiro precisa desempenhar sua função de maneira eficaz, para que a gestante reconheça as ações realizadas pelo mesmo.

Conclui-se que apesar das gestantes reconhecerem a importância das ações realizadas pelo enfermeiro durante o pré-natal é necessário que estes profissionais busquem estratégias 
para melhorar a assistência às gestantes no sentido de reforçar as ações de educação em saúde e a criação de vínculo entre à gestante e o serviço de saúde.

Espera-se que este estudo contribua para ampliação do conhecimento em relação às ações dos enfermeiros frente à condução do acompanhamento pré-natal, encoraje os profissionais a realizar um trabalho eficaz e que possa orientar novos estudos a fim de investigar o trabalho dos enfermeiros em busca de qualificar a assistência à mulher no prénatal.

Como limitação do estudo tem-se o fato de que os resultados apresentados expõem as percepções somente de um dos envolvidos no processo de cuidar no acompanhamento prénatal. Assim, sugere-se que novos estudos sejam realizados no sentido de buscar as percepções dos enfermeiros em relação a seu trabalho, conduta e ações no acompanhamento pré-natal.

\section{REFERÊNCIAS}

ANDRADE, J. C.; GURGGEL, M. D.; SOUZA, R. S.; NORÕES, L. N.; SOUSA, K. M.; SANTOS, M. R. V.; SOUSA, M. A. A percepção das gestantes sobre o pré-natal realizado pelo enfermeiro (a) na estratégia saúde da família do município de Parnaíba. SANARE, v. 14, (supl.) 1, COPISP, p. 127. 2015.

ARAÚJO, D. M. R.; VILARIM, M. M.; SABROZA, A. R.; NARDI, A. E. Depressão no período gestacional e baixo peso ao nascer: uma revisão sistemática da literatura. Cad. Saúde Pública, Rio de Janeiro, v. 26, n. 2, p. 219-227, fev, 2010. Disponível em: http://dx.doi.org/10.1590/S0102-311X2010000200002. Acesso em: set. 2015.

BARDIN, L. Análise de Conteúdo. Lisboa, Portugal; Edições 70, LDA, 2009.

CARRARA, G.L. R.; OLIVEIRA, J. P. Atuação do enfermeiro na educação em saúde durante o pré-natal: uma revisão bibliográfica. Revista FafibeOn-Line. Ano VI, n. 6, p. 96-109, nov. 2013, Disponível em: http://www.unifafibe.com.br/revistasonline/arquivos/revistafafibeonline/sumario/28/11122013185545.pdf. Acesso em: set. 2015.

CARVALHO, J. L. A visão das gestantes sobre as ações educativas do enfermeiro- uma breve aproximação. Especialização em atenção básica em Saúde da Família. 2010, p. 01-23. Universidade Federal de Minas Gerais, UFMG. Campos Gerais, 2010.

CUNHA, M. A.; MAMEDE, M. V.; DOTTO, L. M. G.; MAMEDE, F. V. Assistência pré-natal: competências essenciais desempenhadas por enfermeiros. Revista de Enfermagem, Rio Branco, v. 13, n. 1, p. 146-153, jan./mar. 2009. Disponível em: http://dx.doi.org/10.1590/S1414-81452009000100020. Acesso em: out. 2015.

DIAS, E. G.; SANTO, F. G. E.; SANTOS, I. G. R.; ALVES, J. C. S.; SANTOS, T. M. F. Percepção das gestantes quanto a importância das ações educativas promovida pelo enfermeiro no pré-natal em um unidade básica de saúde. Revista Eletrônica Gestão \& Saúde. v. 6, n. 3, p. 2695-10. 2015a. Disponível em: http://dx.doi.org/10.18673/gs.v6i3.22431. Acesso em: mai. 2016. 
DIAS, E. G.; ALVES, J. C. S.; VIANA, J. M.; SANTOS, I. M.; SILVA, J. P. Percepções sobre a gravidez em um grupo de adolescentes grávidas do município de Janaúba-MG. Revista Eletrônica Gestão \& Saúde, v. 06, n. 02, p.1239-53, 2015b. Disponível em: http://dx.doi.org/10.18673/gs.v6i2.22466. Acesso em: nov. 2015.

FREITAS, E. S.; BOSCO, S. M. D.; SIPPEL, C. A.; LAZZARETTI, R. K. Recomendações Nutricionais na Gestação. Revista destaques acadêmicos, CCBS/UNIVATES; ano 2, n. 3, 2010.

LEMES, A. G. Assistência de enfermagem a gestante na primeira consulta de pré-natal. Revista Eletrônica da Univar, $\quad$ v. $\quad 1, \quad$ n. $\quad 8, \quad$ p. $\quad 70-73 . \quad 2012 . \quad$ Disponível el http://revista.univar.edu.br/index.php/interdisciplinar/article/view/81. Acesso em: nov. 2016.

MARTINELLI, K. G.; NETO SANTOS, E. T.; GAMA, S. G. N.; OLIVEIRA, A. E. Adequação do processo da assistência pré-natal segundo os critérios do programa de humanização do pré-natal e nascimento e rede cegonha. Rev. Bras. Ginecol. Obstet. Rio de Janeiro, v. 36, n. 2, fev. 2014. Disponível em: http://dx.doi.org/10.1590/S010072032014000200003. Acesso em: mai. 2016.

MARTINS, J. S. A.; DANTAS, F. A.; ALMEIDA, T. F.; SANTOS, M. B. R. A Assistência de Enfermagem no Pré-Natal: Enfoque na Estratégia da Saúde da Família. Revista UNIABEU, Belford Roxo, v. 5, n. 9, jan./abr., p. 278-288, 2012.

MOREIRA, M. A.; CARVALHO, L. L.; RIBEIRO, P. S. Percepção de gestantes sobre a atuação da enfermeira na assistência pré-natal: estudo analítico. Arq. Ciênc. Saúde. v. 23, n. 1, p. 78-82, jan./mar., 2016.

NIQUINI, R. P.; BITTENCOURT, S. A.; LACERDA, E. M. A.; SAUNDERS, C.; LEAL, M. C. Avaliação do processo da assistência nutricional no pré-natal em sete Unidades de Saúde da Família do Município do Rio de Janeiro. Ciência \& Saúde Coletiva, v. 17, n. 10, p. 2805-2816, 2012. Disponível em: http://dx.doi.org/10.1590/S141381232012001000028. Acesso em: mai. 2016.

NUNES, D. C.; SILVA, L. A. M. Humanização na assistência de Enfermagem durante gestação, parto e puerpério e seus desafios na promoção de saúde. Revista Mineira de Ciências da Saúde, v. 4, p. 57-68, set. 2012.

PARIS, G. F.; PELLOSO, S. M.; MARTINS, P. M. Qualidade da assistência pré-natal nos serviços públicos e privados. Rev Bras Ginecol Obstet. v. 35, n. 10, p. 447-52, 2013.

SALES, K. C.; SEMENTE, P. S. N.; FERNANDES. E. R. L. Cartão da Gestante: um elo fundamental. Revista Brasileira de Informações Científicas. v. 4, n. 3, p. 8-15. Jul./set. 2013.

SILVA, M. Z. N.; ANDRADE, A. B.; BOSI, M. L. M. Acesso e acolhimento no cuidado pré-natal à luz de experiências de gestantes na Atenção Básica. Saúde Debate, Rio de Janeiro, v. 38, n. 103, p. 805-816, out./dez. 2014. Disponível em: http://dx.doi.org/10.5935/0103-1104.20140073. Acesso em: nov. 2016.

SOUSA, A. J. C. Q.; MENDONÇA, A. E. O.; TORRES, G. V. Atuação do enfermeiro no pré-natal de baixo risco em uma unidade básica de saúde. Carpe Diem: Revista Cultura e Científica do UNIFACEX. v. 10, n.10, p. 1- 15, 2012. Acesso em: set. 2015.

SOUZA, B. C., BERNARDO, A. R. C., SANTANA, L. S. O Papel do Enfermeiro no Pré-Natal Realizado no Programa de Saúde da Família - PSF. Interfaces Científicas - Saúde e Ambiente, Aracaju v. 2 n.1 p. 83-94 out. 2013. Disponível em: http://dx.doi.org/10.17564/2316-3798.2013v2n1p83-94. Acesso em: jun. 2016.

SOUZA, V. B.; ROECKER, S., MARCON, S. S. Educação em Saúde na Assistência Pré-Natal: Percepção de Gestantes Atendidas na Rede Básica de Maringá-PR. III Semana de Enfermagem em HUOP. XIX Jornada de Cascavelense de Enfermagem. UNIOESTE. Campus de Cascavel. 10-12 maio, 2010.

SPINDOLA, T.; PROGIANTI, J. M.; PENNA, L. H. G. Opinião das gestantes sobre acompanhamento da enfermeira obstetra no pré-natal de um hospital universitário. Cienc Enferm. v. 18, n. 2, p. 65-73, 2012.

VIEIRA, B. D.; PARIZOTTO, A. P. A. V. Alterações psicológicas decorrentes do período gravídico, Unoesc \& Ciência - ACBS, Joaçaba, v. 4, n. 1, p. 79-90, jan./jun. 2013. 